\title{
Validez de la Escala de Evaluación Cognitiva de Montreal (MoCA) para determinar deterioro cognitivo en pacientes con esquizofrenia
}

\author{
Lorena Rodríguez-Bores Ramírez, ${ }^{1}$ Ricardo Saracco-Álvarez, ${ }^{1}$ \\ Raúl Escamilla-Orozco, ${ }^{1}$ Ana Fresán Orellana ${ }^{2}$
}

Artículo original

\section{SUMMARY}

Background

Schizophrenia is a severe mental disorder characterized by complex symptoms and impaired cognitive function, which has been considered a core feature. Research into cognition and schizophrenia over the past years has taken several different approaches including psychometric assessments.

\section{Objective}

The Montreal Cognitive Assessment (MoCA) has been proven useful in detecting cognitive impairment in patients with several forms of dementia. Our aim was to assess its validity as a screening instrument for cognitive impairment in schizophrenic patients.

\section{Material and methods}

One hundred patients with diagnosis of schizophrenia were recruited at the Schizophrenia Clinic of the National Institute of Psychiatry in Mexico. The MoCA and MMSE were used to assess cognitive functions in these patients. For the concurrent validity, the MoCA, MMSE and PANSS were used, and existing methods were utilized to determine reliability and validation.

\section{Results}

According to the MMSE-education adjusted cut-off scores, only $8 \%$ of the patients in our sample were found with cognitive impairment in contrast with $69 \%$ of the total score obtained from the MoCA. The MoCA was tested for reliability with a Cronbach's alpha $=0.71$ similar to the one reported for MMSE $(\alpha=0.70)$ showing appropriate concurrent validity.

\section{Conclusions}

The MoCA is a cognitive tool with adequate psychometric properties as a screening instrument for the detection of mild cognitive impairment in schizophrenia. It also promises to fill an urgent unmet need for a brief screening tool capable of detecting patients with $\mathrm{MCl}$ and schizophrenia.

Key words: Mild cognitive impairment, schizophrenia, Montreal Cognitive Assessment Scale.

\section{RESUMEN}

\section{Introducción}

La esquizofrenia es un trastorno mental que posee múltiples dimensiones sintomáticas y las alteraciones cognitivas son características cardinales en la evolución del trastorno. El uso de clinimetría para evaluar dichas deficiencias puede aportar información en la investigación en este campo.

\section{Objetivo}

Determinar la validez y confiabilidad de la Escala de Evaluación Cognitiva Montreal (MoCA) para la evaluación del déficit cognitivo en pacientes con el diagnóstico de esquizofrenia.

\section{Material y métodos}

Se evaluaron cien pacientes con diagnóstico de esquizofrenia reclutados en la Clínica de Esquizofrenia del Instituto Nacional de Psiquiatría Ramón de la Fuente Muñiz. Se aplicaron las escalas de MoCA, MMSE y PANSS para la valoración de las funciones cognitivas. Se estableció la confiabilidad del MoCA con el Alfa de Cronbach y se estableció su validez concurrente con el MMSE y la subescala cognitiva de la PANSS.

\section{Resultados}

De acuerdo al MMSE (con ajuste de escolaridad) el $8 \%$ de los pacientes fueron clasificados con deterioro cognitivo en contraste con el puntaje total del MoCA que reporto $69 \%$ de la muestra con deterioro cognitivo. La escala MoCA obtuvo una confiabilidad (Alfa de Cronbach $=0.71)$ similar a la observada por el MMSE $(\alpha=0.70)$ y adecuada validez concurrente.

\section{Conclusiones}

La MoCA es un instrumento confiable y válido para establecer la presencia de deterioro cognitivo leve en pacientes con esquizofrenia, sencillo y de fácil aplicación en la práctica clínica psiquiátrica cotidiana.

Palabras clave: Esquizofrenia, deterioro cognitivo leve, Escala de evaluación cognitiva de Montreal.

Subdirección de Servicios Clínicos, Instituto Nacional de Psiquiatría Ramón de la Fuente Muñiz (INPRFM).

Subdirección de Investigaciones Clínicas, INPRFM.

Correspondencia: Dra. Ana Fresán Orellana. Subdirección de Investigaciones Clínicas, Instituto Nacional de Psiquiatría Ramón de la Fuente Muñiz. Calz. México-Xochimilco 101, San Lorenzo Huipulco, Tlalpan, 14370, México DF. E-mail: fresan@imp.edu.mx

Recibido primera versión: 5 de agosto de 2014. Segunda versión: 23 de septiembre de 2014. Aceptado: 6 de noviembre de 2014 


\section{INTRODUCCIÓN}

Entre los trastornos psiquiátricos, la esquizofrenia se caracteriza por ser un trastorno con dimensiones sintomáticas complejas. El pronóstico es pobre debido al deterioro en múltiples funciones cognitivas lo que dificulta una adecuada adaptación social, académica y laboral. ${ }^{1,2}$ El deterioro cognitivo se refiere a la pérdida de funciones cognitivas, específicamente memoria, atención y velocidad de procesamiento de la información. El inicio y los cambios que conlleva pueden observarse como un deterioro lento y gradual que, en muchas ocasiones, comienza desde antes del primer episodio psicótico. $^{3-5}$ Además se ha observado que dicho deterioro progresa a lo largo de los estados clínicos, tanto psicóticos como en remisión, a pesar del tratamiento e incluso antes de la aparición de los síntomas psicóticos. ${ }^{6} \mathrm{El}$ alcance de los déficits neurocognitivos en la esquizofrenia es muy amplio y se ha reportado que, en contraste con el rendimiento estándar de la población general, ${ }^{5}$ los pacientes con esquizofrenia presentan múltiples fallas en diferentes áreas tales como la atención, la memoria, el procesamiento del lenguaje, la función ejecutiva, la percepción, la capacidad motora y la inteligencia general. ${ }^{7}$

Debido a que el deterioro cognitivo contribuye al desajuste social y laboral de estos pacientes, se hace necesaria una reflexión sobre el tema de su evaluación. El uso clínico de escalas y pruebas puede ser de utilidad, por lo que es necesario contar con pruebas clinimétricas que sean fáciles de aplicar, rápidas, confiables y válidas.

El Examen Cognoscitivo Breve (MMSE por sus siglas en inglés) es un instrumento de fácil aplicación, rápido y ampliamente utilizado para el escrutinio del deterioro cognitivo en pacientes con demencia. ${ }^{8-11}$ Por otro lado, se ha reportado que el MMSE es inadecuado para identificar déficits cognitivos incipientes o complejos. ${ }^{12,13}$ La escala de síntomas positivos y negativos PANSS, utilizada para evaluar la severidad de los síntomas en la esquizofrenia, posee un factor para evaluar la dimensión cognitiva. ${ }^{14}$ En estudios de validación de esta escala, se han encontrado correlaciones entre el factor cognitivo y el desempeño en pruebas neuropsicológicas, concluyendo que el componente cognitivo de la PANSS puede ser una medida válida para los déficits cognitivos en la esquizofrénica. ${ }^{15,16}$ Sin embargo, a pesar de que la estimación del factor cognitivo de la PANSS puede proveer información útil cuando la valoración neuropsicológica completa no sea posible en el escenario clínico cotidiano, estudios de validación muestran que no es recomendable o adecuada para sustituir otras evaluaciones para el deterioro cognitivo en la esquizofrenia. ${ }^{17}$

Otro instrumento breve, de fácil aplicación, que ha mostrado una adecuada capacidad para la detección del deterioro cognitivo es la Escala de Evaluación Cognitiva de Montreal (MoCA por sus siglas en inglés). Este instrumento fue desarrollado para la detección de deterioro cognitivo en pacientes que presentan un perfil cognitivo complejo, como el deterioro cognitivo leve (determinado cuando existe una declinación de las funciones mentales pero que no cumple criterios para considerarse demencia), incluyendo reactivos que exigen mayor estabilidad cognitiva para calificarse como normales, sobre todo en el área de las funciones ejecutivas y la evocación de memoria. ${ }^{13}$ Este instrumento ha sido ampliamente utilizado para la detección del deterioro cognitivo leve en población de pacientes con Alzheimer y ha sido validado en múltiples entidades neuropsiquiátricas y cerebrovasculares. ${ }^{12,18-20}$ Así mismo ha mostrado mayor sensibilidad para la detección del deterioro cognitivo comparado con el MMSE en pacientes geriátricos y con demencia. En cuanto al uso de la MoCA en la esquizofrenia, existe limitada información acerca de su validación ${ }^{21,22}$ y los resultados reportados requieren ser ampliados.

Por lo anterior, el objetivo del presente estudio fue obtener los datos de validación de la MoCA para la evaluación del deterioro cognitivo en pacientes mexicanos con el diagnóstico de esquizofrenia.

\section{MATERIAL Y MÉTODO}

\section{Participantes}

Se reclutaron pacientes de ambos sexos, de 18 a 45 años de edad, quienes recibían atención en la Clínica de Esquizofrenia, con diagnóstico clínico previamente establecido de esquizofrenia de cualquier tipo, de acuerdo a los criterios del DSM-IV-TR, ${ }^{23}$ con menos de diez años de evolución desde el establecimiento del diagnóstico, sin presencia de episodio agudo de psicosis (PANSS 60-120 puntos) que impidiera la aplicación de los instrumentos al momento de la evaluación. Se excluyeron a todos aquellos que presentaran trastornos afectivos concomitantes, trastornos psicóticos secundarios a alguna causa médica o secundarios al uso y/o abuso de sustancias.

Se realizó una evaluación transversal que incluyó la recolección de datos socio-demográficos y clínicos del padecimiento mediante un formato diseñado con este propósito a través de la entrevista directa con el paciente y sus familiares.

Este estudio fue aprobado por los Comités de Ética y de Investigación del Instituto Nacional de Psiquiatría Ramón de la Fuente Muñiz. La participación de los sujetos fue voluntaria, se proporcionó la información en un proceso de consentimiento informado y la identificación de los pacientes fue conservada como información confidencial.

\section{Descripción de las escalas e instrumentos de evaluación}

Examen Cognoscitivo Breve (Mini Mental State ExaminationMMSE). El MMSE es la escala más ampliamente utilizada para evaluar el deterioro cognitivo. ${ }^{8}$ El puntaje máximo es un total de 30 puntos y los reactivos están agrupados en cinco apartados que evalúan la orientación, la memoria in- 
mediata, la atención y el cálculo, el recuerdo diferido, el lenguaje y la construcción. El punto de corte para el deterioro cognitivo se establece habitualmente en 24 puntos. Si hay que anular algún punto (a causa de analfabetismo, ceguera, hemiplejia) se hace un nuevo cálculo proporcional utilizando una tabla de ajuste que proporciona datos de referencia en función de la edad y del nivel educativo, y que puede servir para comparar los resultados de un paciente. ${ }^{9}$ Los estudios de validación en pacientes con Alzheimer demuestran que esta escala tiene una buena sensibilidad (87-100\%) y especificidad (62-100\%) para detectar el deterioro cognitivo. ${ }^{9-11}$

Escala de síntomas positivos y negativos (PANSS). La escala de síntomas positivos y negativos (PANSS) es un instrumento específico para la esquizofrenia y valora tres principales áreas: evalúa el síndrome positivo, negativo y la psicopatología general desde un punto de vista dimensional. ${ }^{24}$ Estudios recientes han presentado evidencia a favor de cinco áreas de la sintomatología (positivo, negativo, cognitivo, ansiedad/ depresión y excitación). ${ }^{25} \mathrm{El}$ modelo pentagonal ha sido validado en pacientes mexicanos con esquizofrenia permitiendo dilucidar los diversos aspectos clínicos de la enfermedad. ${ }^{26}$

Escala de la evaluación cognitiva de Montreal (MoCA). Esta prueba evalúa la función ejecutiva y visoespacial, la identificación, la memoria, la atención, el lenguaje, la abstracción, el recuerdo y la orientación; con un tiempo de aplicación de cinco a 10 minutos, en versiones traducidas a más de 10 idiomas. La especificidad de la MoCA para excluir controles normales fue buena: $87 \%$. Se ha estimado la sensibilidad de la MoCA como excelente $(90 \%)$ para detectar deterioro cognitivo leve y es considerablemente más sensible que el MMSE. 12,13 El valor del punto de corte para determinar un deterioro cognitivo es de $>26$ puntos.

\section{Análisis estadístico}

La descripción de las características clínicas y demográficas se realizó con frecuencias y porcentajes para las variables categóricas y con medias y desviaciones estándar (D.E.) para las variables continuas. Para determinar la validez concurrente, se determinó la asociación lineal con el coeficiente de correlación de Pearson entre la puntuación total de la escala MoCA con la puntuación total del MMSE y el puntaje de la subescala cognitiva de la PANSS. La confiabilidad de la MoCA fue valorada mediante el alfa de Cronbach para obtener la consistencia interna del instrumento. El nivel de significancia estadística se estableció con un valor $\mathrm{p}<0.05$.

\section{RESULTADOS}

\section{Características demográficas de la muestra}

Se incluyeron un total de 100 pacientes con el diagnóstico de esquizofrenia que acudieron de forma consecutiva al servi- cio de la Clínica de Esquizofrenia del Instituto Nacional de Psiquiatría Ramón de la Fuente Muñiz.

De los pacientes incluidos, $65 \%$ fueron hombres y $35 \%$ fueron mujeres, con una edad promedio de $34.1 \pm 8.7$ años (rango 18-53 años) y una escolaridad de $11.7 \pm 3.1$ años (rango cuatro a 20 años), equivalente al segundo año de bachillerato. En relación al estado civil, el $83 \%$ se encontraba sin pareja al momento del estudio, el 14\% se encontraba casado y el 3\% restante separados o divorciados. El 54\% reportó un bajo nivel socioeconómico, seguido por el $45 \%$ con un nivel socioeconómico medio y sólo un paciente $(1 \%)$ reportó un alto nivel socioeconómico. Del total de los pacientes, el $43 \%$ se encontraba desempleado, el $25 \%$ se dedicaba a labores en el hogar, el $9 \%$ eran estudiantes y el $23 \%$ contaba con un trabajo remunerado.

\section{Características clínicas de la muestra}

El 93\% de los pacientes tuvieron el diagnóstico de esquizofrenia paranoide con una edad de inicio del padecimiento reportada por los pacientes de $23.6 \pm 7.4$ años, con una duración de la psicosis no tratada (DPNT) promedio de 139.5 \pm 146.3 semanas (mediana=104 semanas), equivalente a más de dos años con presencia de francos síntomas psicóticos sin haber recibido tratamiento especializado. El $48 \%$ de los pacientes reportó haber sido hospitalizado en algún momento a lo largo del estudio, teniendo un promedio de $1.9 \pm 1.7$ hospitalizaciones psiquiátricas (rango 1-8 hospitalizaciones) con un promedio de estancia intrahospitalaria de $8.9 \pm 9.6$ semanas (rango 1-52 semanas), siendo la edad promedio de la primera hospitalización a los $25.9 \pm 7.7$ años (rango 15-50 años).

El espectro de la psicopatología de los pacientes con esquizofrenia incluidos en el estudio fue evaluada mediante la PANSS de cinco factores. Los promedios en las puntuaciones de la PANSS, así como las puntuaciones promedio de la MoCA y el MMSE, se muestran en el cuadro 1.

Cuadro 1. Gravedad sintomática y evaluación cognitiva de la muestra

\begin{tabular}{lcc}
\hline Variable & Media & $\begin{array}{c}\text { Desviación } \\
\text { estándar }\end{array}$ \\
\hline Gravedad sintomática (PANSS) & & \\
- PANSS positivo & 19.2 & 5.6 \\
- PANSS negativo & 22.6 & 5.7 \\
- PANSS cognitivo & 18.3 & 5.4 \\
- PANSS excitabilidad & 5.8 & 2.3 \\
- PANSS depresión/ansiedad & 9.6 & 4.0 \\
- PANSS total & 75.7 & 15.9 \\
Evaluación cognitiva & & \\
- MoCa total & 23.0 & 3.9 \\
- MMSE total & 27.6 & 2.5 \\
\hline
\end{tabular}




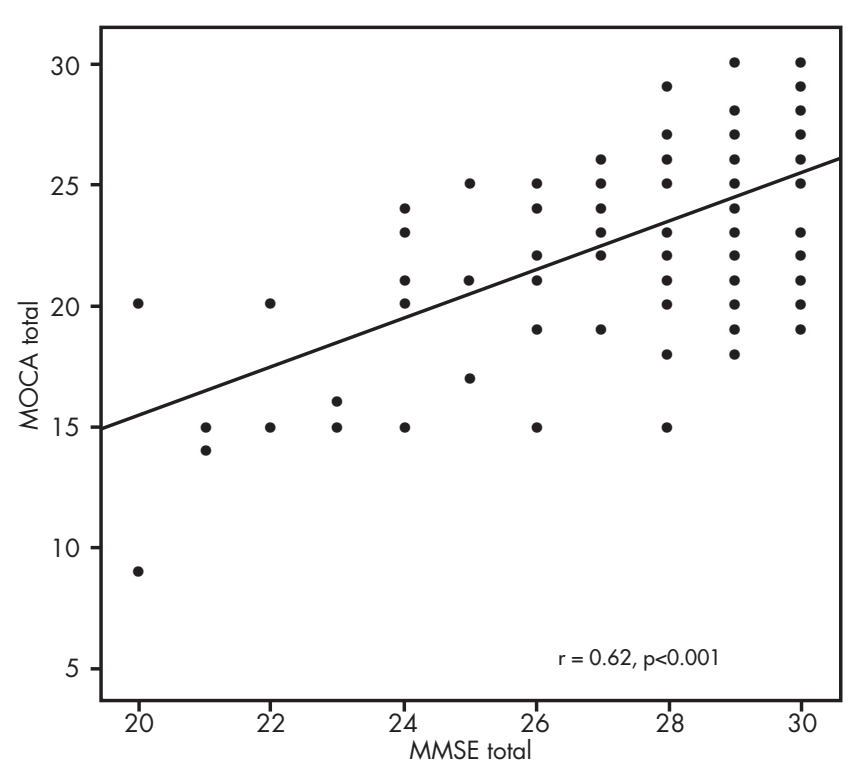

Figura 1. Correlación de la puntuación total de la MoCA y el puntaje total del MMSE.

\section{Deterioro cognitivo}

De acuerdo a los ajustes realizados a la puntuación del MMSE en relación con la escolaridad de los pacientes, tan sólo el 8\% de los pacientes fueron clasificados con deterioro cognitivo. En relación a la MoCA, y de acuerdo al punto de corte sugerido por los autores del instrumento (punto de corte de 26) y el ajuste realizado por la escolaridad de los sujetos (incremento de un punto en la calificación total cuando la escolaridad es <12 años), el 69\% de los pacientes presentó un deterioro cognitivo.

\section{Confiabilidad y validez concurrente de la MoCA en pacientes con esquizofrenia}

La Escala de Evaluación Cognitiva de Montreal obtuvo un alfa de Cronbach de 0.71 , indicativo de una moderada confiabilidad, similar a la observada por el MMSE, el cual fue de alfa $=0.70$. Se obtuvo una elevada correlación $(r=0.62$, $\mathrm{p}<0.001$ ) entre los puntajes totales de la MoCA y el MMSE (figura 1) así como con la subescala cognitiva de la PANSS (figura 2), indicativo de la adecuada validez concurrente de la MoCA para la evaluación del deterioro cognitivo.

\section{DISCUSIÓN}

Se ha descrito que las funciones cognitivas de los pacientes con esquizofrenia se ven afectadas a lo largo de la enfermedad. La búsqueda de factores evaluadores de los cambios en las funciones cognitivas sigue siendo una premisa en la investigación clínica de los mismos. Varios informes han descrito la estrecha relación entre la función cognitiva y los

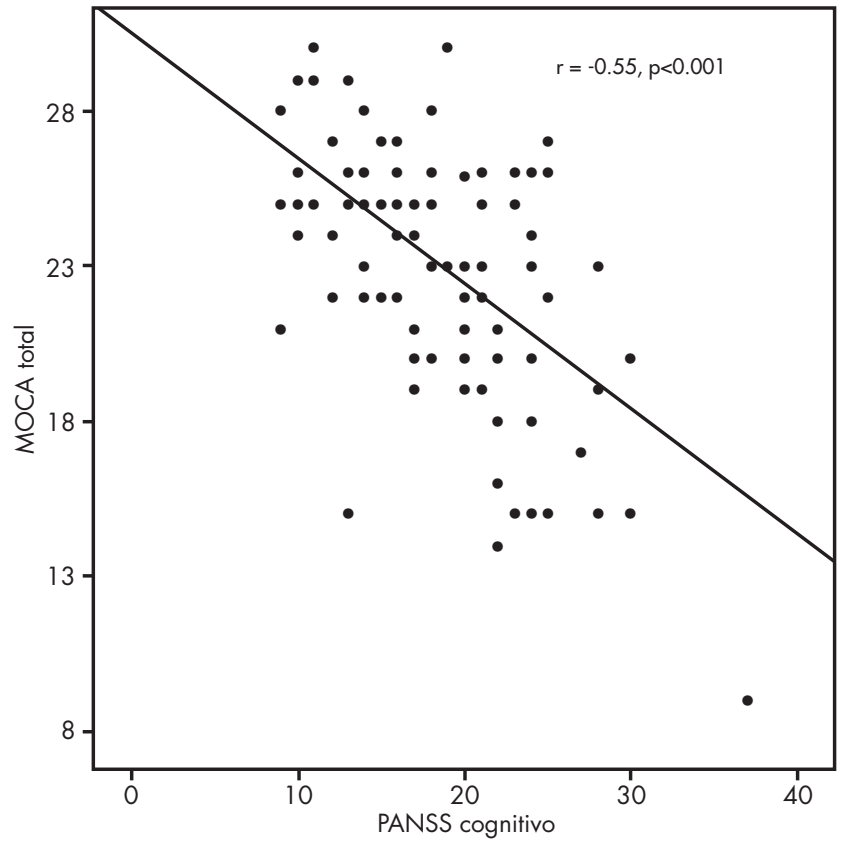

Figura 2. Correlación del puntaje total de la MoCA y la subescala cognitiva de la PANSS.

déficits sociales en la esquizofrenia. Esos déficits en las funciones limitan su desempeño en todas las áreas de su vida y cada vez hay más pruebas de que la cognición social en la esquizofrenia está relacionada con las marcadas disfunciones sociales que presenta, incluso tras controlar el rendimiento en tareas neurocognitivas. ${ }^{27}$

Al revisar las características demográficas de nuestra muestra, se observó que la mayoría de los pacientes no mantenía ninguna relación de pareja, lo que coincide con otros reportes donde se ha descrito una correlación importante entre la cantidad y calidad de las relaciones sociales reales, y el estado cognitivo. A lo largo de varios estudios ${ }^{28}$ todos los constructos neurocognitivos fundamentales (principalmente la memoria inmediata, la secundaria, y las funciones ejecutivas) mostraron correlaciones significativas con el resultado funcional social. Más de la mitad de la muestra se encontraba sin actividad laboral remunerada al momento del estudio; los déficits neurocognitivos también se correlacionan con los déficits en la ejecución de habilidades específicas que son críticas para llevar una vida independiente y productiva. ${ }^{29}$ En algunos estudios que evalúan la relación entre el funcionamiento cognitivo y la situación laboral, han considerado al déficit cognitivo como una característica nuclear del trastorno que afecta desde la restricción de las relaciones sociales hasta la reducción de la probabilidad de tener y mantener un empleo. La DPNT registrada en nuestro estudio fue menor a la reportada en otras muestras. ${ }^{30}$ Existe una gran variabilidad en torno a este fenómeno, ya que algunos pacientes pueden acudir a recibir atención en semanas o meses posteriores al inicio de 
los primeros síntomas psicóticos o pueden permanecer sin ser atendidos durante años.

El objetivo central del presente estudio fue el obtener los datos de confiabilidad y validez de la MoCA en un grupo de pacientes con esquizofrenia. El perfil del déficit cognoscitivo observado al emplear tanto el MMSE como la MoCA fue ampliamente heterogéneo. Al valorar la muestra con ambas escalas, se reportó una menor puntuación promedio al utilizar la escala MoCA que con el MMSE, con sus respectivos ajustes de acuerdo a la escolaridad, detectándose más casos de deterioro cognitivo leve al utilizar MoCA en contraste con los detectados con el MMSE. Parece ser que la sensibilidad de la MoCA es mayor para la detección del deterioro cognitivo en contraste con aquel que puede ser detectado mediante el MMSE.

Algunos autores han definido al deterioro cognitivo como aquella queja subjetiva de pérdida de memoria, que también puede ser reconocida por un familiar, pero se dificulta su detección en quienes la función cognitiva general y las actividades de la vida diaria permanecen intactas y cuyas pruebas neuropsicológicas presentan puntuaciones con 1.5 desviaciones estándar por debajo de la media, ajustada para edad y escolaridad. ${ }^{31}$ Debido a lo anterior, las diferencias encontradas en torno al número de pacientes en esta muestra con deterioro cognitivo pueden ser explicadas debido a que el MMSE es un instrumento que detecta mayores alteraciones cognitivas mientras que la MoCA tiene la capacidad para detectar variantes del funcionamiento cognitivo que no afectan la funcionalidad de los pacientes y es capaz de detectar etapas tempranas o incipientes del deterioro. De forma adicional, para el caso específico de la esquizofrenia habría otras variables que pudieran estar incluidas de forma indirecta en el deterioro cognitivo observado, tales como el coeficiente intelectual (CI) premórbido bajo, asociado a la esquizofrenia. ${ }^{32}$

La MoCA, como instrumento cognitivo de tamizaje, mostró una adecuada validez concurrente al ser comparada tanto con los puntajes obtenidos con el MMSE como con la gravedad global observada en la sintomatología cognitiva evaluada por la PANSS, lo que es consistente con lo reportado en otros estudios. ${ }^{33-35}$ La gravedad del cuadro sindromático en los pacientes no es estable a lo largo del tiempo, por lo que aun cuando se observa una adecuada validez concurrente de la MoCA con la subescala cognitiva de la PANSS, será necesario determinar si ésta, al igual que la PANSS, es sensible a los probables cambios cognitivos observados a lo largo del padecimiento.

No obstante, la consistencia interna de la MoCA resultó con un alfa de Cronbach de 0.70 lo que nos indica que la escala cuenta con una confiabilidad moderada para la detección de deterioro cognitivo en pacientes con esquizofrenia. Aun cuando se considera que una escala es confiable cuando presenta un alfa superior a 0.80 , es oportuno comentar que no se cuenta con datos publicados de la validez y confia- bilidad de la escala MoCA en población abierta mexicana y que será necesario contar con futuros estudios que evalúen su consistencia interna en poblaciones similares, incluyendo las variables confusoras mencionadas con anterioridad para poder estimar con mayor certeza si la MoCA es un adecuado instrumento de tamizaje para la valoración del deterioro cognitivo leve en pacientes con esquizofrenia.

En general, y a pesar de las limitaciones mencionadas con respecto a la difícil evaluación del deterioro cognitivo en los pacientes con esquizofrenia, los resultados del presente estudio muestran que la MoCA puede ser un instrumento efectivo para la realización del tamizaje del deterioro cognitivo en los pacientes con esquizofrenia y que, en el caso de que sus propiedades clinimétricas sean replicadas e incluso mejoradas en futuros estudios, permitirá tener un mayor conocimiento acerca de este importante factor asociado al padecimiento. De esta forma, se contará con una herramienta válida y breve que le permitirá al clínico incluir estrategias de rehabilitación cognitiva en el plan de tratamiento del paciente con esquizofrenia.

\section{REFERENCIAS}

1. Rössler W, Salize HJ, van Os J, Riecher-Rössler A. Size of burden of schizophrenia and psychotic disorders. Eur Neuropsychopharmacol 2005;15(4):399-409.

2. Kessler RC, Aguilar-Gaxiola S, Alonso J, Chatterji S et al. The global burden of mental disorders: an update from the WHO World Mental Health (WMH) surveys. Epidemiol Psichiatr Soc 2009;18(1):23-33.

3. Malla AK, Norman RM, Joober R. First-episode psychosis, early intervention, and outcome: what have we learned? Can J Psychiatry 2005;50(14):881-891.

4. Black K, Peters L, Rui Q, Milliken $H$ et al. Duration of untreated psychosis predicts treatment outcome in an early psychosis program. Schizophr Res 2001;47(2-3):215-222.

5. Stirling J, White C, Lewis S, Hopkins R et al. Neurocognitive function and outcome in first episode schizophrenia: a 10-year follow-up of an epidemiological cohort. Schizophr Res 2003;65:75-86.

6. Galinska B, Szulc A, Czernikiewicz A. Duration of untreated psychosis in first-episode schizophrenia: clinical and cognitive correlates. Psychiatr Pol 2005;39(5):859-868.

7. Harvey PD, Geyer MA, Robbins TW, Krystal JH. Cognition in schizophrenia: from basic science to clinical treatment. Psychopharmacology (Berl) 2003;169(3-4):213-214.

8. Folstein M, Folstein S, MCHugh PR. Minimental State: a practical method for grading cognitive state of the patients for the clinican. J Psychiatr Res 1975;12:189-198.

9. Crum M, Anthony JC, Bassett SS, Folstein MF. Population-based norms for the Mini-Mental State Examination by age and educational level. J American Medical Association 1993;269(18):2386-239.

10. Thal LT, Grundmen M, Golden R. Alzheimer diseases: a correlational analyses of the Blessed information memory concentration test and the Minimental State. Exam Neurology 1986;36:262-264.

11. Feher EP. Establishing the limits of the Minimental State examination of substensts. Arch Neurol 1992;49:87-92.

12. Pendlebury ST, Cuthbertson FC, Welch SJV, Mehta Z, Underestimation of cognitive impairment by Mini-Mental State Examination versus the Montreal Cognitive Assessment in patients with transient ischemic attack and stroke: a population-based study. Stroke 2010;41:1290-1293. 
13. Nasreddine ZS, Phillips NA, Bédirian V, Charbonneau $S$ et al. The Montreal Cognitive Assessment, MoCA: a brief screening tool for mild cognitive impairment. J Am Geriatr Soc 2005;53(4):695-699.

14. Berman I, Viegner B, Merson A, Allan E et al. Differential relationships between positive and negative symptoms and neuropsychological deficits in schizophrenia. Schizophr Res 1997;25(1):1-10.

15. Hofer B, Niedermayer G, Kemmler MA, Rettenbacher E et al. Trebo, C.G. Widschwendter, W.W. Fleischhacker Cognitive impairment in schizophrenia: clinical ratings are not a suitable alternative to neuropsychological testing. Schizophr Res 2007;126-131.

16. Cameron AM, Oram J, Geffen GM, Kavanagh DJ et al. Working memory correlates of three symptom clusters in schizophrenia. Psychiatry Res 2002;110(1):49-61.

17. Rodríguez-Jiménez R, Bagney A, Mezquita L, Martínez-Gras I et al. PARG. Cognition and the five-factor model of the positive and negative syndrome scale in schizophrenia. Schizophr Res 2013;143(1):77-83.

18. Dalrymple-Alford JC, MacAskill MR, Nakas CT, Livingston L et al. The MoCA: well-suited screen for cognitive impairment in Parkinson disease. Neurology 2010;(19):1717-725.

19. Roalf DR, Moberg PJ, Xie SX, Wolk DA et al. Comparative accuracies of two common screening instruments for classification of Alzheimer's disease, mild cognitive impairment, and healthy aging. Alzheimers Dement 2013;(5):529-537.

20. Yokomizo JE, Simon SS, De Campos Bottino CM. Cognitive screening for dementia in primary care: a systematic review. Int Psychogeriatr 2014;15:1-22.

21. Fisekovic S, Memic A, Pasalic A. Correlation between MoCA and mmse for the assessment of cognition in schizophrenia. Acta Inform Med 2012;20(3):186-189.

22. Arunpongpaisal S, Sangsirilak A. Using MoCA-Thai to evaluate cognitive impairment in patients with schizophrenia. J Med Assoc Thai 2013;96(7):860-865.

23. American Psychiatric Association (APA). Manual Diagnóstico y Estadístico de los Trastornos Mentales DSM-IV-tr. Barcelona: Masson; 2002.

24. Kay S, Fisbein A, Opler L. The Positive and Negative Syndrome Scale (PANSS) for schizophrenia. Schizophr Bull 1987;13:261-276.
25. Lancon C, Auqioer P, Nayt G, Reine G. 2000. Stability of the five-factor structure of the Positive and Negative syndrome Scale (PANSS). Schizophr Res 2002;42:231-239.

26. Fresán A, De la Fuente-Sandoval C, Loyzaga C, García-Anaya M et al. A forced five-dimensional factor analysis and concurrent validity of the Positive and Negative Syndrome Scale in Mexican schizophrenic patients. Schizophr Res 2005;72(2-3):123-129.

27. Green MF, Kern RS, Braff DL, Mintz J. Neurocognitive deficits and functional outcome in schizophrenia: are we measuring the "right stuff"? Schizophr Bull 2000;26(1):119-136.

28. Ziv I, Leiser D, Levine J. Social cognition in schizophrenia: cognitive and affective factors. Cogn Neuropsychiatry 2011;16(1):71-91.

29. Evans JD, Heaton RK, Paulsen JS, Palmer BW et al. The relationship of neuropsychological abilities to specific domains of functional capacity in older schizophrenia patients. Biol Psychiatry 2003;53(5):422-430.

30. Apiquián-Guitart R, Fresán-Orellana A, García-Anaya M, LóyzagaMendoza $\mathrm{C}$ et al. Impacto de la duración de la psicosis no tratada en pacientes con primer episodio psicótico. Estudio de seguimiento a un año. Gac Méd Méx 2000;142(2).

31. Petersen RC, Doody R, Kurz A, Mohs RC et al. Current concepts in mild cognitive impairment. Arch Neurol 2001;58(12):1985-92.

32. Woodberry KA, Giuliano AJ et al. Premorbid IQ in schizophrenia: A meta-analytic review. Am J Psychiatry 2008;165:579-587.

33. Duro D, Simões MR, Ponciano E, Santana I. Validation studies of the Portuguese experimental version of the Montreal Cognitive Assessment (MoCA): confirmatory factor analysis. J Neurol 2010;257(5):728734.

34. Pendlebury ST, Cuthbertson FC, Welch SJ, Mehta Z et al. Underestimation of cognitive impairment by mini-mental state examination versus the montreal cognitive assessment in patients with transient ischemic attack and stroke: a population-based study. Stroke 2010;(6):1290-1293.

35. Dalrymple-Alford JC, MacAskill MR, Nakas CT, Livingston L et al. The MoCA: well-suited screen for cognitive impairment in Parkinson disease. Neurology 2010;75(19):1717-1725.

Artículo sin conflicto de intereses 